\title{
Crystal truncation rods in kinematical and dynamical x-ray diffraction theories
}

\author{
Vladimir M. Kaganer \\ Paul-Drude-Institut für Festkörperelektronik, Hausvogteiplatz 5-7, D-10117 Berlin, Germany
}

(Dated: February 8, 2020)

\begin{abstract}
Crystal truncation rods calculated in the kinematical approximation are shown to quantitatively agree with the sum of the diffracted waves obtained in the two-beam dynamical calculations for different reflections along the rod. The choice and the number of these reflections are specified. The agreement extends down to at least $\sim 10^{-7}$ of the peak intensity. For lower intensities, the accuracy of dynamical calculations is limited by truncation of the electron density at a mathematically planar surface, arising from the Fourier series expansion of the crystal polarizability.
\end{abstract}

PACS numbers: 61.10.Dp, 61.10.Nz, 61.10.Kw, 68.05.Cf

The planar surface of a semi-infinite crystal gives rise to a diffraction pattern consisting of lines normal to the surface and passing through the bulk diffraction peaks. The intensity distribution along these lines, called crystal truncation rods (CTRs), contains information on the surface structure.1,2 CTR calculations, commonly performed in the kinematical (single scattering) approximation, are the basis of x-ray surface crystallography $\underline{3,4,5,6}$ The high-intensity CTR regions close to the Bragg reflections that require dynamical (multiple scattering) calculations are not sensitive to surface structure and are usually excluded from considerations in the surface structure determination studies.

On the other hand, many studies of crystalline films and multilayers are based on dynamical diffraction theory and are mostly restricted to the close vicinity of the Bragg peaks $\stackrel{7,8,9}{ }$ The substrate peak position is commonly used as a reference to study the film relaxation. The intensity of the substrate peak can also be used as a reference to obtain the film structure factor on an absolute scale to determine, e.g., ordering in sublattices 10 The thinner the film, the larger the part of the CTR that needs to be analyzed. Studies of very thin (a few atomic layers) films require the analysis of the whole CTR. The problem of an accurate calculation of the CTR intensity in the whole wave vector range has been the subject of a number of investigations, $11,12,13,14,15,16,17,18,19$ but a conclusive recipe has not been given yet. It is well established that the two-beam dynamical diffraction theory is very accurate in the vicinity of the respective Bragg reflection but fails in the middle between two reflections. The resolution of the latter problem was attempted by appropriate multibeam calculations. ${ }^{12,16,17}$ Recently, Pavlov et $a l .19$ proposed to calculate the diffracted intensity in a wide angular range by summing up the amplitudes of the diffracted waves for different reflections, each amplitude being the solution of the corresponding two-beam diffraction problem.

In the present paper, I show that the sum of the solutions of the two-beam diffraction problems for the reflections along a CTR coincides with the kinematical formula. The number and the choice of reflections that have to be included in the summation are specified. Dynamical and kinematical calculations disagree in the close vicinity of the Bragg reflections where the dynamical solution is correct while the kinematical one diverges, and in the regions where the diffracted intensity is less than $\sim 10^{-7}$ of the peak intensity. In this latter region, the dynamical calculations are less accurate. The error in the dynamical calculations originates from the Fourier expansion of the electron density over the reciprocal lattice vectors, so that it cannot be remedied by a more accurate multibeam dynamical theory.

The analysis is based on the equality

$$
\frac{i F_{h k L}}{\exp (2 \pi i L)-1}=\frac{1}{2 \pi} \sum_{l=-\infty}^{\infty} \frac{F_{h k l}}{L-l} .
$$

It is derived below and the limits of its validity are established. Here $h k l$ are the integer Miller indices of the reflections ( $h$ and $k$ correspond to the directions in the surface plane and $l$ is along the surface normal), $L$ is the continuous coordinate along a crystal truncation rod ( $L=q a / 2 \pi$, where $q$ is the $z$-component of the momentum transfer and $a$ is the lattice spacing in $z$-direction), and $F_{h k L}=\sum_{j} f_{j} \exp \left[2 \pi i\left(h x_{j}+k y_{j}+L z_{j}\right) / a\right]$ is the structure factor of the unit cell (the sum is taken over all atoms in the unit cell, $f_{j}$ is the atomic scattering factor of the $j$ th atom and $x_{j}, y_{j}, z_{j}$ are its coordinates). It will be shown below that Eq. (11) is valid if and only if the electron density obtained by the back Fourier transform of $F_{h k l}$ is entirely contained inside the unit cell. This latter requirement results in the cutting of the atomic electron densities at the surface.

The left-hand side of Eq. (1) is proportional to the scattering amplitude of a semi-infinite crystal in the kinematical approximation $1,2,3,4,5$ The kinematical theory for semi-infinite crystals $\frac{9}{-}$ provides the remaining prefactors. The amplitude of the scattered wave is

$$
E^{\mathrm{kin}}(h k L)=\frac{\lambda r_{e}}{a^{2} \gamma_{\text {out }}} \frac{i F_{h k L}}{\exp (2 \pi i L)-1} .
$$

Here $\lambda$ is the wavelength, $r_{e}$ is the classical radius of the electron, and $\gamma_{\text {out }}=\sin \Phi_{\text {out }}$, where $\Phi_{\text {out }}$ is the angle between the diffracted wave and the crystal surface. A similar factor for the incidence wave, $\gamma_{\text {in }}=\sin \Phi_{\text {in }}$, where $\Phi_{\text {in }}$ is the angle between the incident beam and the sur- 
face, will be used later. The amplitude of the incident wave is taken equal to 1 .

Each term of the sum on the right side of Eq. (1), after having been multiplied by the same factor $\lambda r_{e} /\left(a^{2} \gamma_{\text {out }}\right)$, is the asymptotic expression of the two-beam dynamical diffraction theory for angular deviations from the Bragg peak that are much larger than the dynamical peak width. Within dynamical theory, the amplitude of the diffracted wave at large deviations from the Bragg peak for a given reflection $\mathbf{H}$ is given by $E_{\mathbf{H}}=\chi_{\mathbf{H}} /\left[2 \gamma_{\text {out }}\left(\gamma_{\text {in }}+\right.\right.$ $\left.\gamma_{\text {out }}+\psi_{\mathbf{H}}\right)$ ], where $\chi_{\mathbf{H}}$ is the Fourier component of the polarizability and $\psi_{\mathbf{H}}=H_{z} / \kappa$ (here $\kappa=2 \pi / \lambda$ is the wave vector in vacuum). The position along the CTR $L=(a / \lambda)\left(\gamma_{\text {in }}+\gamma_{\text {out }}\right)$ is expressed through the incidence and the exit angles, while the position of the Bragg peak on the CTR is $l=-(a / \lambda) \psi_{\mathbf{H}}$. Substitution of these expressions gives $E_{\mathbf{H}}=\lambda r_{e} F_{\mathbf{H}} /\left[2 \pi a^{2} \gamma_{\text {out }}(L-l)\right]$, which is equal to the corresponding term in Eq. (11) multiplied by the factor given above. Then, the sum of the amplitudes of diffracted waves calculated in the two-beam dynamical theory

$$
E^{\mathrm{dyn}}(h k L)=\sum_{l} E_{h k l}^{\mathrm{dyn}}
$$

for all reflections $h k l$ along a CTR $h k$ coincides with the kinematical solution. For non-grazing incidence and exit, the resulting amplitude depends on the $z$-component of momentum transfer $q=2 \pi L / a$, rather than the incident and the exit angles $\Phi_{\text {in }}$ and $\Phi_{\text {out }}$ separately. Pavlov et $a l \stackrel{19}{n}$ have arrived to an equivalent formula by solving the Takagi-Taupin equations.

In the close vicinity of each reflection $h k l$, for angular deviations comparable to the Darwin width, the amplitudes of the other reflections are much smaller (on the order of the polarizability $\chi \sim 10^{-5}$ ) than that of the actual reflection, and Eq. (3) can be applied, keeping in mind that all other terms can be neglected compared to $E_{h k l}^{\mathrm{dyn}}$. At larger angular deviations, Eq. (3) is the first order (in $\chi$ ) approximate solution of the multibeam dynamical diffraction problem that includes all Bragg reflections along a given CTR. Such an approximation is valid since at most one Bragg reflection occurs at once at the diffraction condition when moving along the CTR. Hence, Eq. (3) describes the intensity distribution along a CTR around Bragg peaks and far from them, down to intensities $\sim \chi$ of the peak intensity, which covers almost the whole CTR. Eq. (3) fails at the order of $\chi^{2}$. Before ascertaining the problems arising at such low intensities, let us consider the CTR calculations based on Eq. (3).

I have implemented the dynamical diffraction equations in the formulation of Stepanov et al ${ }^{20}$ Since Eq. (3) involves the summation of the scattering amplitudes, a proper treatment of the phases of the Fourier components $\chi_{h k l}$ of the polarizability is essential. The structure factor calculations require the origin of the unit cell to be chosen once for all reflections. This is not so in the programs by Sergey Stepanov 21 that choose the origin separately for each reflection to minimize the phase of $\chi_{h k l}$. Such a choice is correct as long as a single reflection is involved in the calculations. When working with the amplitudes of several reflections, Eq. (3), this is not appropriate. Also, for layered structures, the interference pattern depends on the relative displacements of the crystal lattices of different layers, which requires a common origin for the calculation of all $\chi_{h k l}$ 's 10 The requirement of a common origin is usual in surface $\mathrm{x}$-ray structure analysis but not appreciated in the dynamical diffraction calculations.

Equally important, but probably less evident, is the choice of the atomic positions in the unit cell with respect to the surface. The surface is commonly taken at $z=0$, and one of the atoms is placed at the origin. If so, the surface cuts half of this atom (and other atoms at the same level), which leads to wrong CTR intensities. For example, with the surface at $z=0$, the Ga and As atoms in the bulk GaAs unit cell cannot be placed at the depths $0,1 / 4,1 / 2,3 / 4$. Rather, they have to be put at the depths $1 / 8,3 / 8,5 / 8,7 / 8$. Only this choice leads to an agreement between dynamical and kinematical calculations. Note that this choice of the atomic positions is irrelevant to the kinematical formula (2), since the kinematical calculation, based on the summation over atoms and unit cells, contains only one structure factor $F_{h k L}$ (with the continuous parameter $L$ ), the phase of which is lost when calculating the intensities. In contrast, the relative phases of the structure factors $F_{h k l}$ (with integer $l$ 's) depend on the choice of atomic positions.

The choice of the reflections that have to be included in the sum (3) follows from Eq. (11). Since $L$ starts from zero, positive and negative $l$ 's are equally important. Hence, the sum should contain the solutions of the twobeam dynamical problems in both reflection (the Bragg case) and transmission (the Laue case). Although the dynamical diffraction equations are formulated in Ref. 20 for the reflection case, they do not require any change to include the transmission case, since the diffracted wave is anyway directed back into vacuum. In other words, the reflections that formally correspond to the Laue case (the diffracted wave propagates into the crystal when the Bragg condition is satisfied) become the Bragg case reflections because the deviation from the Bragg condition is so large that the diffracted wave propagates into the vacuum. The number of equations to be included in the sum (11) or (3) depends on the decay of the structure factors $F_{h k l}$ with increasing $l$. The atomic scattering factors $f(s)$ are decreasing functions of $s=\sin \theta / \lambda$, where $\theta$ is the Bragg angle of the respective reflection, with $s \approx 2 \AA^{-1}$ as a characteristic scale for this decay. Then, for reflections with $l \gg h, k$ the lattice spacing is $d=a / l$ and, from Bragg's law $s=1 / 2 d$, reflections up to $l_{\max } \approx 4 a$ have to be included (here $a$ is in angstroms). Hence, a reasonable estimate is $l_{\max } \approx 25$. In fact, the calculations below show that $l_{\max } \approx 10$ is sufficient for practical calculations.

Figure 1(a) compares kinematical and dynamical calculations of the CTR $11 L$ from a hypothetical bulk- 

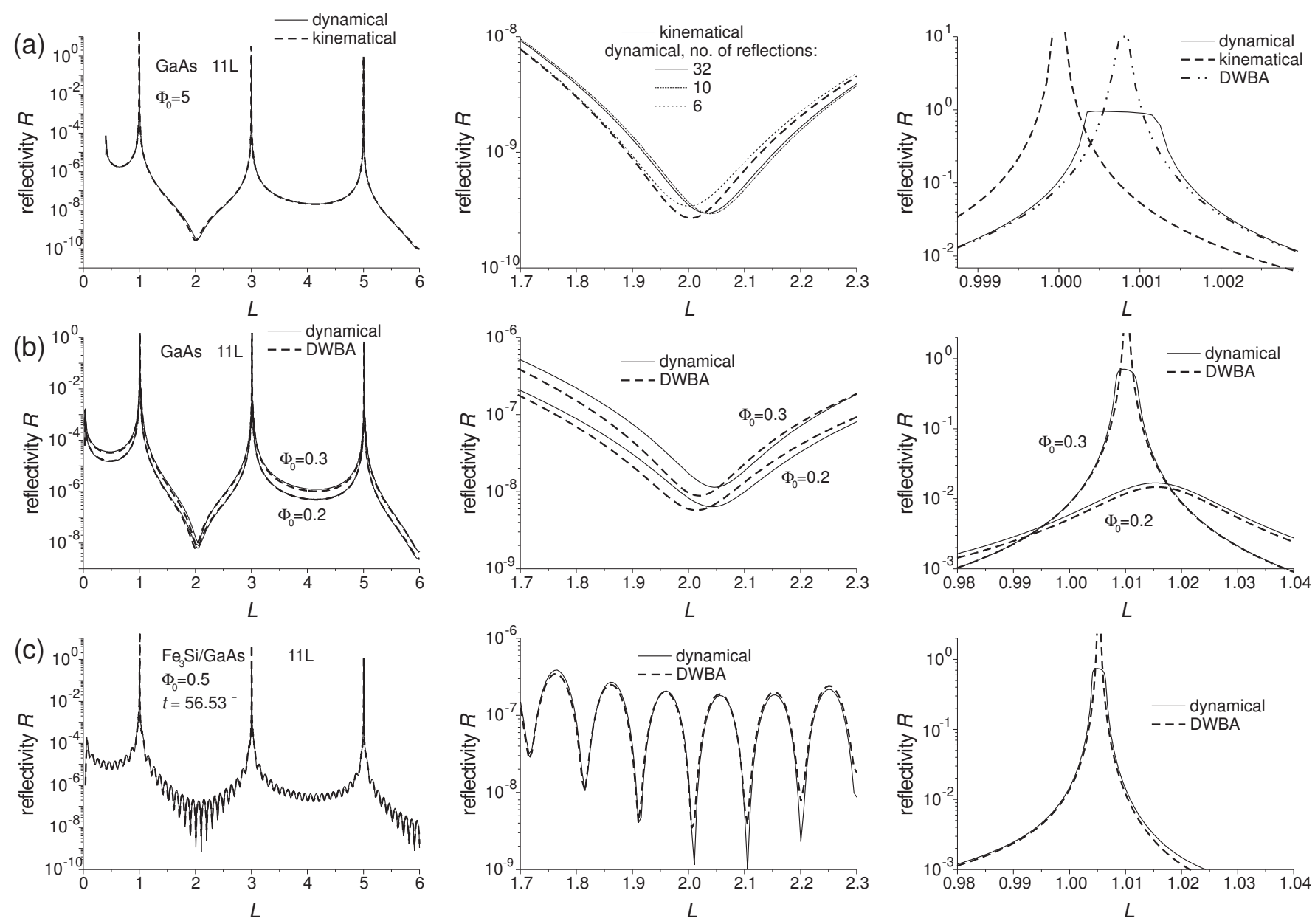

FIG. 1: Crystal truncation rods $11 L$ from a bulk-terminated GaAs $(001)$ crystal at (a) non-grazing incidence angle $\Phi_{0}=5^{\circ}$ and (b) grazing incidence angles $\Phi_{0}=0.3^{\circ}$ and $\Phi_{0}=0.2^{\circ}$, above and below the critical angle $\alpha_{c}=0.24^{\circ}$. (c) CTR $11 L$ from a $56.53 \AA$ thick $\mathrm{Fe}_{3} \mathrm{Si}$ film on $\mathrm{GaAs}(001)$ at an incidence angle $\Phi_{0}=0.5^{\circ}$. The two right panels magnify the curves of the left panel at the intensity minimum (bulk-forbidden reflection 112) and close to the maximum (bulk reflection 111). Dynamical, kinematical, and distorted-wave Born approximations are compared.

terminated $\operatorname{GaAs}(001)$ crystal. The reflectivities $R=$ $\left(\gamma_{\text {out }} / \gamma_{\text {in }}\right)|E(h k L)|^{2}$ are plotted. A non-grazing incidence angle $\Phi_{0}=5^{\circ}$ is chosen to ensure kinematical diffraction conditions. In the left panel, presenting the whole CTR, the two calculated curves are almost indistinguishable. At the 111 bulk reflection (the right panel) the kinematical curve is slightly shifted with respect to the dynamical one because of refraction at the surface, and diverges at the Bragg position. A small but important discrepancy between the curves is revealed in the middle panel that enlarges the region of the intensity minimum, close to the bulk-forbidden reflection 112. Dynamical calculations are performed with different numbers of terms in the sum (3). One can see from the plot that 10 terms (i.e., the sum of two-beam dynamical calculations for the reflections $11 l$ with odd $l$ in the range $-9 \leq l \leq 9)$ are sufficient for the convergence of the series. However, the sum (3) does not converge to the kinematical solution even if the number of the involved reflec- tions is increased further. The origin of the disagreement is in the Fourier expansion of the electron density over the reciprocal lattice vectors $h k l$. This expansion is certainly correct for an infinite bulk crystal that consists of a periodic repetition of the unit cells. However, if a unit cell is cut out of the crystal, its electron density does not coincide with the sum of electron densities of the atoms that belong to this unit cell. Figure 2 illustrates this statement. Here the atoms of the GaAs bulk structure are represented by balls with radii equal to their covalent radii. The broken line selects one unit cell. Its position is chosen to minimize the overlap of the electron densities belonging to the atoms of different unit cells. Still, parts of the electron density of atoms of the chosen unit cell are out of that unit cell, and parts of atoms from neighboring unit cells fall into the chosen unit cell. These do not affect the bulk structure factor calculations, since the unit cells are periodically repeated. However, the dynamical Bragg diffraction from a semi-infinite crystal implies a 


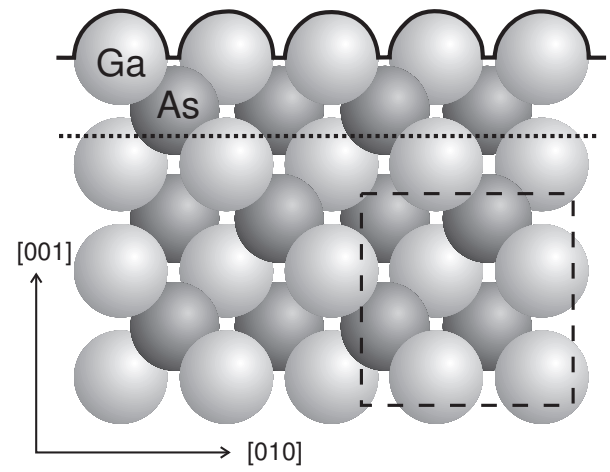

FIG. 2: View of a truncated GaAs bulk structure in the [100] direction. Atoms are represented by balls with their radii equal to the covalent radius of the respective element. A unit cell marked by broken lines is chosen to minimize the overlap of the electron densities from atoms of different unit cells. The dotted straight line represents a cut by a mathematically planar (001) surface. The solid line is a physical (001) surface terminated by atoms.

truncation of the sum over the unit cells, which is equivalent to a rigid truncation of the electron density by a plane (dotted line in Fig. 2). Such a cut of the electron density of an infinite crystal by a plane removes parts of the top atoms and artificially adds the parts of the atoms belonging to the next layer. In contrast, the physical surface is rough on that scale since it contains the entire densities of the atoms of the top layer, as shown by the thick line in Fig. 2 ,

The difference in these electron densities does not play a role in the vicinity of Bragg peaks, where many atoms contribute to diffraction, but becomes essential between Bragg peaks, where the surface atoms provide the main contribution to the diffracted wave. To provide an additional proof that this effect is the only source of discrepancy between the dynamical and kinematical calculations, I have repeated the same calculations for artificial atoms two times smaller in size placed in the same lattice, Fig. 3. Such a crystal is not, in fact, physical, since the long-range attractive forces between atoms are not compensated by the short-range repulsion when the distances between atoms exceed the sum of their covalent radii by a factor of 2 . The atomic scattering factors for the artificial atoms $\mathrm{Xa}$ and $\mathrm{Xb}$ are chosen to be Gaussians with widths approximately two times larger than the $f(s)$ functions for Ga and As atoms, Fig. 3(b). The convergence of sum (11) or (3) is fairly slow, Fig. 3(c). The sum of dynamical amplitudes converge to the distorted-wave Born approximation, described below, when 20 terms are included. Each term is a solution of the two-beam dynamical diffraction problem for a reflection $11 l$ with odd $l$ from -17 to +21 .

This result discourages other improvements of the dynamical theory, in particular a complete solution of the multibeam diffraction problem, that could improve the accuracy compared to Eq. (3). As long as a Fourier ex- pansion over reciprocal lattice vectors is employed, the cut of the electron density shown in Fig. 2 persists and does not provide an accuracy that is better than given by Eq. (3), i.e., an accuracy of the order of $\sim 10^{-7}$ with respect to the peak intensity. Fortunately, lower intensities quite rarely arise in experimental CTR studies and are additionally masked by surface reconstruction and roughness.

Equation (3) can be directly applied to grazing incidence diffraction with the same reasoning as above: in the vicinity of a Bragg peak, corrections due to other reflections are negligible, and in the remaining part of a CTR the sum (3) is the first order (over $\chi$ ) perturbation solution of the multibeam diffraction problem. The kinematical approximation is extended, for the grazing incidence diffraction case, as the distorted-wave Born approximation (DWBA) $\stackrel{9,22,23,24}{9}$ The DWBA formulation in terms of the reciprocity theorem in electrodynamics is most straightforward ${ }^{23,24}$ In the zeroth order, the scattering problem is solved for a uniform medium having the same polarizability $\chi_{0}$ as the crystal under investigation. The scattering problem is solved twice, with the waves incident on the surface under the incidence angles $\Phi_{\text {in }}$ and $\Phi_{\text {out }}$, respectively. The solution of each problem in the medium consists of two plane waves with the amplitudes $D_{i}(i=1,2)$, corresponding to the transmitted and the specularly reflected waves in vacuum. A convenient way to find these solutions is to reduce the $4 \times 4$ matrices of the dynamical diffraction problem ${ }^{20}$ to $2 \times 2$ matrices. In this way, layered structures with different $\chi_{0}$ 's can easily be treated. Then, the kinematical solution is replaced by

$$
E^{\mathrm{DWBA}}\left(\Phi_{\mathrm{in}}, \Phi_{\text {out }}\right)=\sum_{i, j=1,2} D_{i}^{\mathrm{in}} D_{j}^{\text {out }} E^{\mathrm{kin}}\left(h k L_{i j}\right) .
$$

The superscripts "in" and "out" distinguish the respective zeroth order solutions. The parameters $L_{i j}=$ $(a / \lambda)\left(u_{i}^{\text {in }}+u_{j}^{\text {out }}\right)$ are obtained from the parameters $u_{i}=$ $k_{z i} / \kappa$ describing the wave vectors in the medium (here $\mathbf{k}_{i}$ are the wave vectors of the waves inside the medium). Complex parameters $L_{i j}$ substitute the real parameter $L$ in Eq. (21).

Figure 1(b) compares dynamical and DWBA calculations of the same CTR $11 L$ for the incidence angles below and above the critical angle $\alpha_{c}=0.24^{\circ}$. A good overall agreement is evident from the left panel. The discrepancy at the intensity minimum (middle panel) has the same nature as above. The right panels of Fig. 1(a,b) show that the DWBA and the dynamical calculation agree everywhere except within the Darwin width of the Bragg peak. If the incidence angle is smaller than the critical angle, the results of the two calculations coincide in that region as well.

The extension to layered structures is straightforward. The diffracted beam amplitudes obtained in the twobeam dynamical calculations ${ }^{20}$ are summed up according to Eq. (3). The kinematical amplitude (2) is supplemented by an additional term arising from the finite sum over the layer's unit cells, $F_{h k L}^{\prime}[\exp (2 \pi i N L)-$ 

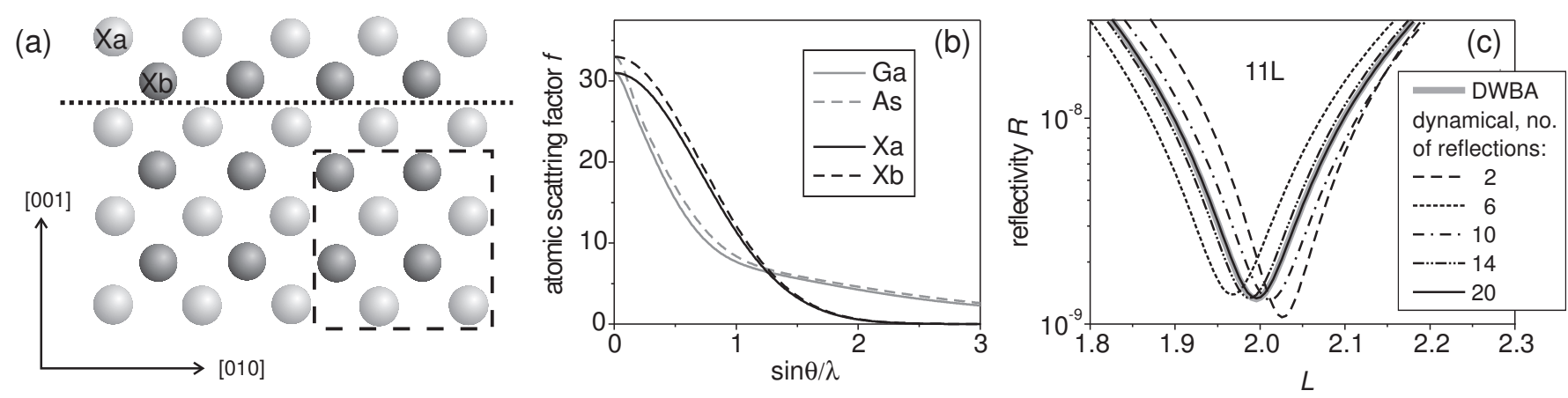

FIG. 3: (a) An artificial crystal XaXb with the same structure and the lattice parameter as GaAs but with atomic sizes that are two times smaller. The unit cell (broken lines) and a planar surface (dotted line) can be cut between atoms. (b) Atomic scattering factors attributed to atoms $\mathrm{Xa}$ and $\mathrm{Xb}$. The functions $f(\sin \theta / \lambda)$ are chosen to be Gaussians with widths approximately two times larger than those of Ga and As. (c) The CTR $11 L$ from the XaXb semi-infinite crystal in the vicinity of $L \approx 2$ calculated within distorted-wave Born approximation (DWBA) and dynamically, by Eq. (3), with different numbers of involved reflections. As the number of reflections is increased, the dynamical curves converge to the DWBA results. The incidence angle is $\Phi_{0}=0.5^{\circ}$.

1]/[exp $(2 \pi i L)-1]$, where $F_{h k L}^{\prime}$ is the structure factor of the layer unit cell, and $\mathrm{Na}$ is the layer thickness. Figure 1(c) compares dynamical and DWBA calculations for a $\mathrm{Fe}_{3} \mathrm{Si}$ film on $\mathrm{GaAs}(001)$. $\mathrm{Fe}_{3} \mathrm{Si}$ possesses a cubic unit cell with the same lattice spacing as GaAs, so that there is no mismatch between the two lattices $\underline{10}$ The incidence angle is taken $\Phi_{0}=0.5^{\circ}$, so that the DWBA corrections to the kinematical formulas are essential. The film is $N=10$ unit cells thick $(N a=56.53 \AA)$. A perfect agreement between the two curves is evident. Even at the intensity minimum (middle panel), the discrepancy between the two calculations is absent. At the bulk reflection 111 (right panel), the DWBA solution diverges and cannot be used within the Darwin width, while the dynamical solution is correct. The dynamical calculations in Figs. $1(b, c)$ were performed by the summation of solutions of 32 two-beam diffraction problems (11l reflections with odd $l$ in the range $-31 \leq l \leq 31$ were calculated).

The CTR $11 L$ analyzed in Fig. 1 shows a larger discrepancy between dynamical and kinematical calculations than other rods from GaAs(001) surface. Figures $4(a, b)$ present two other rods. The difference between the two calculations diminishes for the $1 \overline{3} L$ rod and almost disappears for the $22 \mathrm{~L}$ rod. Further calculations show that the discrepancy decreases for $\mathrm{A}_{\mathrm{III}} \mathrm{B}_{\mathrm{V}}$ crystals consisting of elements of two different periods in the periodic table [e.g., GaSb, see Fig. 4(c)] and increases for lighter elements from the same period [e.g., AlP, see Fig. [4(d)]. The discrepancy remains quite small on the scale of the whole CTR and hardly can have practical implications. Elemental semiconductors show more sharp minima at the bulk-forbidden reflection 112, see Figs. 目(e,f). Similarly to the case of compound semiconductors, silicon as a lighter element shows a larger, as compared to germanium, discrepancy between dynamical and DWBA calculations, see Fig. 4(f).

Equation (11) remains to be derived. Consider an arbitrary complex one-dimensional function $\rho(z)$ that is equal to zero outside a finite interval $[0, a]$. The Fourier integral of the function $\rho(z)$ therefore involves integration over a finite interval:

$$
F(q)=\frac{1}{a} \int_{0}^{a} \rho(z) \exp (i q z) d z .
$$

On the other hand, the function $\rho(z)$ can be periodically repeated along the $z$-axis,

$$
\rho_{\infty}(z)=\sum_{l=-\infty}^{\infty} \rho(z+l a),
$$

and the periodic function $\rho_{\infty}(z)$ can be expanded into the Fourier series:

$$
\rho_{\infty}(z)=\sum_{l=-\infty}^{\infty} F\left(q_{l}\right) \exp \left(-i q_{l} a\right)
$$

where $q_{l}=2 \pi l / a$. Let $\Omega(z)$ be a function equal to one on the interval $[0, a]$ and equal to zero outside this interval. Then, identically

$$
\rho(z)=\rho_{\infty}(z) \Omega(z) .
$$

The Fourier integral of the left-hand side of this equation is equal to $F(q)$. The Fourier integral of the right-hand side is calculated by performing the integration for each term of the sum:

$$
\begin{aligned}
F(q) & =\sum_{l=-\infty}^{\infty} F\left(q_{l}\right) \int_{0}^{a} \exp \left[i\left(q-q_{l}\right) a\right] d z \\
& =[\exp (i q a)-1] \sum_{l=-\infty}^{\infty} \frac{F\left(q_{l}\right)}{i\left(q-q_{l}\right) a} .
\end{aligned}
$$

It is taken into account that $\exp \left(i q_{l} a\right)=1$ since $q_{l}=$ $2 \pi l / a$ with an integer $l$, so that $q_{l} a$ is a multiple of 


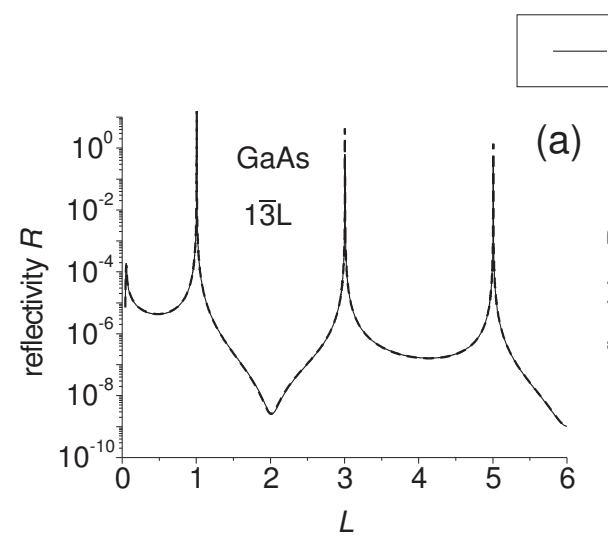

\section{dynamical - - DWBA}
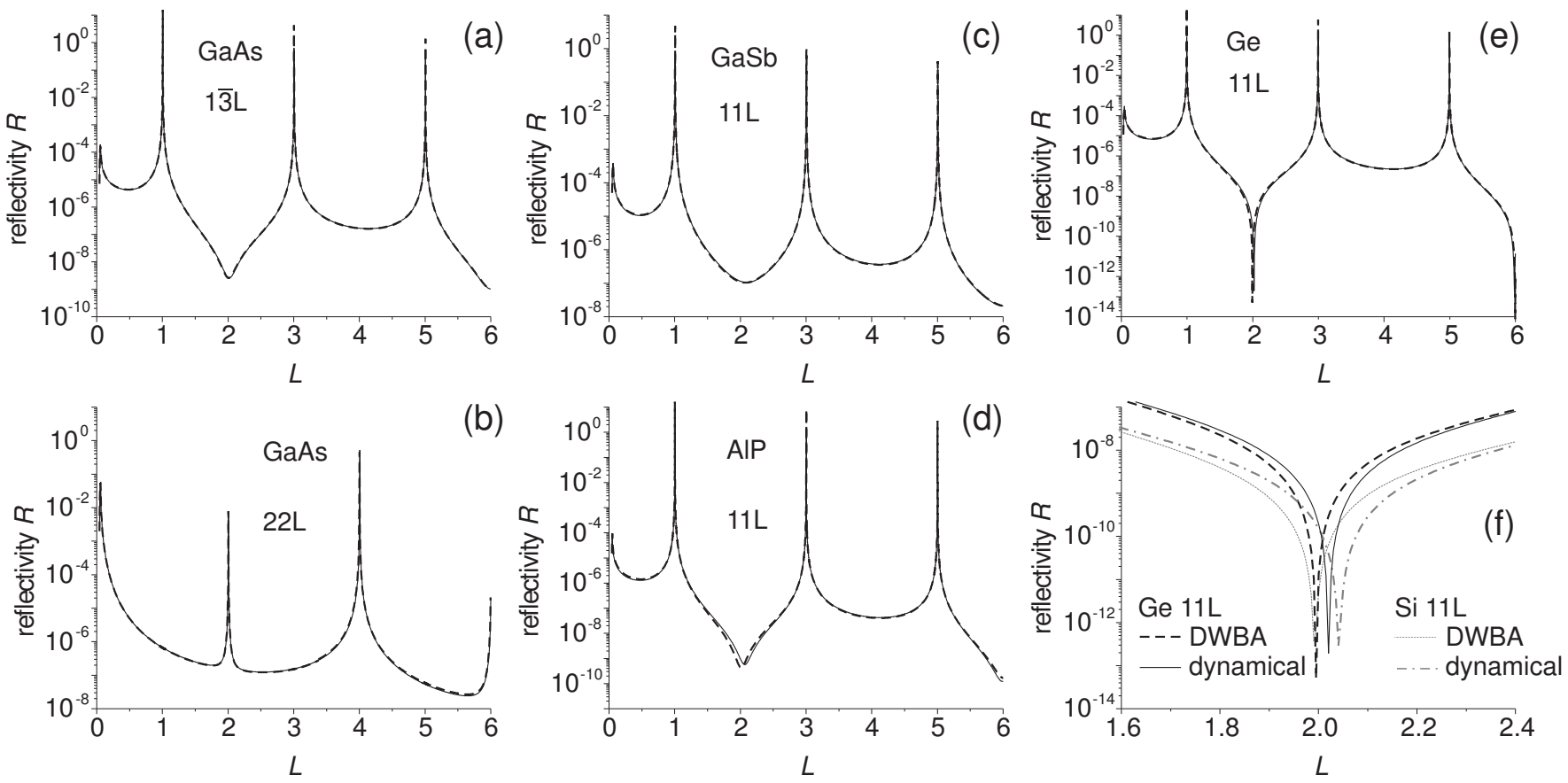

FIG. 4: Crystal truncation rods from GaAs (a,b), other $\mathrm{A}_{\mathrm{III}} \mathrm{B}_{\mathrm{V}}$ compounds (c,d), and elemental semiconductor (e,f) bulktruncated (001) surfaces. Dynamical (full lines) and DWBA (broken lines) calculations are compared. The incidence angle is $\Phi_{0}=0.5^{\circ}$.

$2 \pi$. The function $\rho(z)$ can now be identified with the Fourier component $\rho_{h k}(z)$ in the expansion of the electron density of a one unit cell thick crystalline slab over the wave vectors of its two-dimensional reciprocal lattice. Its Fourier transform $F(q)$ is equal to $F_{h k L}$ with $L=q a / 2 \pi$, and $F\left(q_{l}\right)$ are equal to $F_{h k l}$. Equation (9) reduces to Eq. (11). The crucial point in the derivation is contained in Eq. (8), which explicitly requires that the function $\rho(z)$ is restricted to the interval $[0, a]$.

Hence, the structure factors in Eq. (1) correspond to the electron density constrained to a unit cell cut out of the crystal, rather than to the electron density of the atoms whose centers are inside this unit cell. Parts of the electron density distributions of the atoms belonging to the chosen unit cell are cut away, while parts of the atoms from the surrounding unit cells occur in the chosen unit cell, Fig. 2. The problem can be avoided in rare cases of layered crystals, e.g. graphite, but is enhanced for higher-index surfaces. This effect limits the applicability of the Fourier series expansion of the electron density for the solution of the diffraction problems. The solution of a multibeam diffraction problem instead of Eq. (3), as well as corrections to the dynamical equations that are omitted in Ref. 20, provide improvements of the order of $\chi^{2}$, smaller than the effect of rigid truncation. That is why these corrections are not included in the calculations here. However, only the regions of extremely low intensity are affected. Intensities in these regions are sensitive to surface reconstruction and roughness. Hence, further improvements of the dynamical theory seem of limited practical impact nowadays.

In conclusion, it is shown that the kinematical calculation (or, at small incidence angles, the distorted-wave Born approximation) quantitatively agrees with the sum of the diffracted beam amplitudes obtained in the twobeam dynamical calculation. The number of Bragg reflections that have to be included in the dynamical calculations is estimated from the angular dependence of the atomic scattering factors and amounts to some tens of reflections. Both transmission (Laue) and reflection (Bragg) cases have to be included. The reference unit cell should be chosen to minimize (since it cannot be completely excluded) the cutting of the electron densities of the top atoms by the surface. The agreement between kinematical and dynamical calculations can be lost in two regions. In the Darwin width regions near Bragg reflections the dynamical theory provides correct intensities while the kinematical theory diverges. In the regions of very low intensity (below $\sim 10^{-7}$ of the peak intensity), dynamical theory may fail because of the electron density truncation by a mathematically flat plane instead of the physical surface while the kinematical theory remains applicable.

This work has been inspired by discussions at the 8th Conference on High Resolution X-Ray Diffraction and Imaging (XTOP 2006). 
1 I. K. Robinson, Phys. Rev. B 33, 3830 (1986).

2 E. Vlieg, J. F. van der Veen, S. J. Gurman, C. Norris, and J. E. Macdonald, Surf. Sci. 210, 301 (1989).

3 R. Feidenhans'l, Surface Science Reports 10, 105 (1989).

4 I. K. Robinson, Handbook of Synchrotron Radiation (Elsevier, Amsterdam, 1991), vol. 3, chap. 7, p. 221.

5 I. Robinson and D. Tweet, Rep. Prog. Phys. 55, 599 (1992).

6 E. Vlieg, J. Appl. Cryst. 33, 401 (2000).

7 D. K. Bowen and B. K. Tanner, High Resolution X-Ray Diffractometry and Topography (Taylor \& Francis, London, 1998).

8 P. F. Fewster, X-Ray Scattering from Semiconductors (Imperial College Press, London, 2000).

9 U. Pietsch, V. Holý, and T. Baumbach, High-Resolution X-Ray Scattering: From Thin Films to Lateral Nanostructures (Springer, Berlin, 2004).

10 B. Jenichen, V. M. Kaganer, J. Herfort, D. K. Satapathy, H. P. Schönherr, W. Braun, and K. H. Ploog, Phys. Rev. B 72, 075329 (2005).

11 A. M. Afanas'ev, P. A. Aleksandrov, S. S. Fanchenko, V. A. Chaplanov, and S. S. Yakimov, Acta Cryst. A 42, 116 (1986).

12 R. Colella, Phys. Rev. B 43, 13827 (1991).
13 A. Caticha, Phys. Rev. B 47, 76 (1993).

14 A. Caticha, Phys. Rev. B 49, 33 (1994).

15 T.-S. Gau and S.-L. Chang, Acta Cryst. A 51, 920 (1995).

16 O. Litzman and P. Mikulik, J. Phys.: Condens. Matter 11, 5767 (1999).

17 V. Holý and P. F. Fewster, J. Phys. D: Appl. Phys. 36, A5 (2003).

18 S. G. Podorov, N. N. Faleev, K. M. Pavlov, D. M. Paganin, S. A. Stepanov, and E. Förster, J. Appl. Crystallogr. 39, 652 (2006).

19 K. M. Pavlov, D. M. Paganin, D. J. Vine, and L. Kirste, Phys. Stat. Sol. (a) 00, 00 (2007), in press.

20 S. A. Stepanov, E. A. Kondrashkina, R. Köhler, , D. V. Novikov, G. Materlik, and S. M. Durbin, Phys. Rev. B 57, 4829 (1998).

21 http://sergey.gmca.aps.anl.gov/.

22 S. K. Sinha, E. B. Sirota, S. Garoff, and H. B. Stanley, Phys. Rev. B 38, 2297 (1988).

23 V. E. Dmitrienko and V. M. Kaganer, Metallofizika 9, 71 (1987), [Phys. Metals 9(1), 102 (1990)].

24 V. M. Kaganer, S. A. Stepanov, and R. Köhler, Phys. Rev. B 52, 16369 (1995). 